\title{
Desempenho escolar e gastos municipais por aluno em educação: relação observada em municípios baianos para o ano 2000
}

\section{Resumo}

Este artigo apresenta resultados obtidos para o impacto de recursos educacionais no desempenho escolar de estudantes da $4^{a}$ e da $8^{a}$ séries das redes municipais de municípios baianos. A amostra refere-se aos estudantes matriculados nas séries citadas nas escolas de 274 municípios onde o Governo do Estado da Bahia aplicou testes de rendimento em Português e Matemática no ano de 2000. A análise é feita a partir de regressões múltiplas rodadas no Microsoft Excel 2000, tendo como variável dependente as notas dos alunos e como variáveis de controle um conjunto de proxies para os fatores apontados pela literatura como determinantes do desempenho escolar. Embora os resultados encontrados tenham sido estatisticamente não significativos, a análise desenvolvida busca fornecer parâmetros concretos para uma avaliação objetiva das políticas públicas em educação, em especial no que concerne à eficácia do dispêndio público no ensino fundamental.

Palavras-chave: Eficácia da escola. Recursos escolares. Educação fundamental. Desempenho escolar. Gastos públicos em educação. Economia da Educação.

\footnotetext{
- Agradeço ao Prof. Dr. Robert E. Verhine, da Universidade Federal da Bahia (UFBA), por ler e discutir comigo este artigo ao longo de sua elaboração, e ao Prof. Dr. José Sérgio Gabrielli de Azevedo, atualmente licenciado da UFBA para presidir a Petrobrás, por ter orientado por sete meses o meu trabalho monográfico de conclusão do curso de Economia que deu origem a este artigo. Também meu muito obrigado aos membros da Banca Examinadora (Prof. Dr. Luís Filgueiras, que inclusive assumiu a orientação nos dois últimos meses, após o orientador anterior se licenciar, Prof. Dr. André Garcez Ghirardi, e o então doutorando em Administração Luiz Ricardo Cavalcante) da monografia mencionada, por teceram pertinentes observações que acabaram sendo incorporadas também neste artigo. Agradeço a todos eles por muitos dos meus acertos neste texto - os erros são inteiramente meus.
} 
undertaken by pupils who were enrolled in the $4^{\text {th }}$ and $8^{\text {th }}$ grades in public schools of 274 municipalities throughout the Brazilian State of Bahia during the year 2000. Multiple regressions were observed in Microsoft Excel 2000 using a number of proxies for the factors pointed by the literature as determinants of student achievement, in order to try to explain the marks those pupils got. Although the results have not been statistically significant in any of the regressions, this work is a contribution to provide objective parameters to evaluate public policies in Education in Brazil, especially those concerning the efficiency and effectiveness of the public

expenditure allocated for the elementary public schools.

Keywords: School effectiveness. School resources. Elementary education. Student achievement. Public expenditure in Education. Economics of Education.

\section{Resumen}

\section{Desempeño escolar $y$} gastos municipales por alumno en educación: relación observada en municipios del estado de Bahia para el año 2000

Este artículo presenta resultados obtenidos para el impacto de los recursos escolares sobre el desempeño académico de estudiantes de los años 4 y 8 de escuelas públicas de 274 municipalidades de Bahia, un de los estados federativos de Brasil. A los estudiantes les fue aplicado un teste de rendimiento en Portugués y otro en
Matemática durante el año de 2000. El análisis fue hecha a partir de regresiones múltiplas rodadas en Microsoft Excel 2000, tiendo como variable dependiente los escores obtenidos por los estudiantes y como variables de control un conjunto de proxies para los factores apuntados por la literatura especializada como determinantes del desempeño académico. A pesar de que los resultados obtenidos no fueron estadísticamente significativos, este trabajo busca a fornecer parámetros concretos para una evaluación objetiva de las políticas públicas en educación en Brasil, sobretodo en lo que concierne a la eficacia del dispendio público con las escuelas elementares.

Palabras clave: Eficacia de la escuela. Recursos escolares. Educación elementar. Desempeño estudiantil. Dispendio público en Educación. Economía de la Educación.

\section{Introdução}

A questão da eficácia dos recursos educacionais é um tema que atrai a atenção de estudiosos da educação em todo o mundo. Ainda incipiente no Brasil, essa discussão sob a ótica econômica tem permeado o meio acadêmico de diversos países, sobretudo dos Estados Unidos, há bastante tempo. A questão que se coloca é: os recursos educacionais têm de fato influência na educação de crianças e adolescentes? Em caso afirmativo, qual a melhor forma de empregá-los?

A busca por estas respostas é de grande relevância para os formuladores de políticas públicas em educação e também para os gestores de escolas, sejam estas públicas ou privadas. De todo modo, no 
que tange à educação pública, a constatação ou não da importância dos insumos educacionais na qualidade da educação ofertada pode levar a diferentes maneiras de intervenção do Poder Público no setor.

Este artigo apresenta os resultados de uma pesquisa realizada em 2002, na qual foram utilizados dados de 2000 referentes a gastos municipais por aluno em educação, características socioeconômicas e habilidades iniciais como variáveis explicativas de um modelo econométrico que tem como variável de controle o rendimento, no mesmo ano, em testes de desempenho em Português e Matemática de alunos de $4^{a}$ e $8^{a}$ séries das redes municipais de 274 municípios baianos. O objetivo é buscar verificar o grau de influência, se algum se mostrar relevante, dos recursos educacionais no aprendizado desses estudantes.

Para atingir esse objetivo este artigo parte primeiramente de uma revisão dos resultados que vêem sendo levantados pela literatura econômica internacional sobre o tema, ressaltando, inclusive, a diversidade de conclusões que tem polarizado o debate em duas principais correntes. Em seguida, é apresentado o modelo econométrico aplicado, explicitando a amostra, os dados disponíveis, o método (interseccional) e o processo de escolha das variáveis, pautado na teoria resumida na seção precedente. A seção "Desempenho x recursos...", traz os resultados extraídos da amostra disponível, aduzindo ainda observações metodológicas importantes para o enriquecimento de trabalhos futuros similares. Por fim, a última seção exprime as conclusões a que chegou o trabalho, avaliando até que ponto seus resultados podem ou não atestar o grau de importância dos gastos públicos em educação.

\section{Consensos e dissensos da literatura disponível}

Ao avaliar o impacto dos recursos educacionais no desempenho escolar, o estudioso de Economia da Educação deparase na verdade com dois problemas: se, de fato, tais recursos influenciam a educação e, em caso afirmativo, qual a melhor forma de alocá-los para maximizar a sua influência positiva e minimizar seus custos. As respostas para tais problemas podem variar a depender das características dos alunos, de suas famílias, da escola e da realidade socioeconômica que os circundam.

A literatura econômica que se debruça sobre estas questões utiliza modelos econométricos para avaliar o grau de importância de diversos fatores na educação de crianças e adolescentes. Uma vasta gama de variáveis é escolhida em diversos estudos para verificar quais fatores são mais fortemente correlatos com o desempenho dos estudantes, tais como: o tempo dedicado aos estudos (AKSOY; LINK, 2000), o conteúdo curricular (ALEXANDER, 2000), as instalações físicas da escola (JONES; ZIMMER, 2001), o tamanho da escola (DRISCOLL; HALCOUSSIS; SVORNY, 2003), atenção dada pelos pais ao estudo dos fiIhos (KIM, 2001), a renda familiar (AKSOY; LINK, 2000), características ambientais (MCEWAN, 2003; REES; BREWER; ARGYS, 2000; BETTS; SCHKOLNIK, 2000a e b) e até o grau de sociabilidade dos pais (BUCHEL; DUNCAN, 1998).

Colocando em categorias, os fatores determinantes do desempenho escolar discutidos pela literatura podem ser classificados em três: características socioeconômicas, habilidades pré-adquiridas pelos alunos e recur- 
sos destinados à educação. Unnever, Kerckhoff e Robinson (2000) ilustram bem, no quadro abaixo, os vetores de influência dessas categorias de fatores no rendimento escolar, realçando ainda o efeito da primeira sobre as duas outras categorias, o que exige um cuidado redobrado do pesquisador para isolar as influências indiretas das condições socioeconômicas sobre o desempenho, que podem inflar os parâmetros das habilidades prévias e dos recursos escolares.

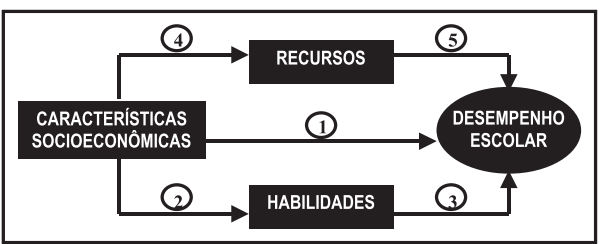

Figura 1 - Relação entre características socioeconômicas, habilidades pré-adquiridas dos estudantes, recursos da escola e desempenho escolar.

Fonte: Adaptado de Unnever, Kerckhoff e Robinson (2000).

Os resultados apontados pela literatura são praticamente unânimes quanto à importância do entorno socioeconômico na educação do estudante. Portanto, as condições da família e do meio social do aluno são de fato responsáveis por uma grande parte do seu desenvolvimento educacional. Famílias estáveis, com bons níveis econômicos, educacionais e culturais, tendem a ter filhos que se dão melhor na escola e futuramente no mercado de trabalho. Assim, para muitos, são os fatores familiares e a influência de grupos sociais que desempenham o papel principal na educação de crianças e adolescentes, cabendo à escola uma participação apenas secundária (MINKEVICH; DAVIS; BASHI, 1980, apud LAVY, 1998). É relativamente pacífica, também, a assertiva de que parte do rendimento do aluno seja explicada pelas habilidades que ele já tenha desenvolvido anteriormente ao ciclo escolar avaliado. No que tange à importância dos recursos educacionais no desempenho escolar, porém, a literatura econômica é divergente.

A corrente majoritária, capitaneada por Eric Hanushek (apud SANDER, 1993; CARD; KRUEGER, 1996; LAVY, 1998; FIGLIO, 1999; ALEXANDER, 2000; UNNEVER; KERCKHOFF; ROBINSON, 2000; AZEVEDO et al., 2002), atualmente professor da Universidade de Stanford, insiste que não há correlação positiva significativa entre os resultados dos estudantes em testes de desempenho e o volume de recursos investidos na escola (HANUSHEK; RIVKIN; TAYLOR, 1996). Para Hanushek, o foco não deveria ser colocar mais dinheiro na escola, mas sim utilizar meIhor os recursos já disponíveis (HANUSHEK, 1994, apud HANUSHEK; RIVKIN; TAYLOR, 1996; LAVY, 1998; FIGLIO, 1999).

Na outra ponta da polêmica, Hedges, Greenwald e Laine (1994, 1996a, 1996b), oriundos da Universidade de Chicago, lideram uma corrente crescente que vê a correlação entre os insumos educacionais e as notas dos estudantes em testes de aproveitamento como grande o suficiente para ser pedagogicamente relevante. Há ainda quem defenda que os recursos educacionais não teriam somente um efeito direto sobre o desempenho dos alunos, mas também um efeito indireto, à medida que podem modificar o comportamento das famílias, tal como, por exemplo, uma eventual redução do tempo que os pais destinam a acompanhar a evolução de aprendizado dos filhos, por acreditarem que escolas com maiores e melhores recursos possam substituir esse papel parental (KIM, 2001). 
Em verdade, o que se constata a partir da leitura dos estudos existentes é que as conclusões acerca da influência dos insumos educacionais na qualidade da educação podem divergir a depender da metodologia e da amostra utilizadas (NASCIMENTO, 2003). A região ou país de origem da amostra, por exemplo, podem significar impactos diferentes dos recursos escolares na década de 70 o impacto positivo desses recursos sobre o desempenho estava associado a sistemas educacionais de países em desenvolvimento, onde predominavam estudantes de baixo status socioeconômico, enquanto que em países desenvolvidos tal influência parecia não existir ou ser irrelevante (HEYNEMAN; LOXLEY, 1982, 1983), mas vinte anos depois esse efeito parecia já ter desaparecido e o impacto dos recursos escolares sobre o desempenho de alunos de países em desenvolvimento parecia ter-se tornado tão reduzido quanto o que Hanushek encontrava para países desenvolvidos (BAKER; GOESLING; LETENDRE, 2002), embora Hedges, Laine e Greenwald (1994, 1996a, 1996b), tenham alertado, desde meados da década de 90, a partir de meta-análises ${ }^{1}$, para o papel significativo de insumos escolares no desempenho estudantil, mesmo em países da OCDE.

Vale ressaltar, contudo, que as conclusões de uma e outra corrente não são necessariamente opostas: a realidade de cada escola e de seus alunos indicará a pertinência ou não de se investir mais em recursos educacionais (como defendem Hedges, Greenwald e Laine), sendo sempre adequada a discussão em torno da eficiência e eficácia dos gastos da escola (ponto central de Hanushek).

\section{Metodologia}

\section{Amostra e número de observações}

Os resultados doravante apresentados partem de uma amostra inicial de 274 dos 417 municípios baianos ${ }^{2}$, nos quais foram aplicados, no ano 2000, pelo Governo do Estado da Bahia, testes de rendimento em Português e Matemática a alunos de $4^{a}$ e de $8^{a}$ séries das redes municipal e estadual. As regressões foram rodadas com o uso do programa de computador Microsoft Excel 2000, que dispõe das ferramentas necessárias para o uso demandado.

Como o foco do trabalho ora apresentado fora sobre as redes municipais de ensino, foi descartado da análise o desempenho dos estudantes de escolas estaduais. Este procedimento reduziu o número de algumas observações, em especial para a $8^{a}$ série, pois havia muitos casos de municípios que não dispunham de rede municipal de ensino, ou só ofertavam vagas até a $4^{a}$ série, sendo a demanda restante suprida pela rede estadual ou pelas redes de municípios circunvizinhos.

Outra carência que reduziu o número de observações foi a ausência de dados. Nem todos os municípios contavam com dados atualizados para todas as variáveis levantadas para o modelo aplicado. Toda vez que isto ocorreu, uma observação (município) foi excluída do modelo. O caso de maior destaque foi Salvador,

\footnotetext{
1 Para tal análise, os autores fizeram uso do teste qui-quadrado inverso, também conhecido como teste de Fischer ou teste P? de Pearson, para verificar a significância média dos coeficientes dos estudos por ele revisados.

${ }^{2}$ Os motivos que levaram o Governo do Estado da Bahia a aplicar os testes de rendimento em escolas de apenas 274 municípios, e não em todos os 417 que integram o Estado, bem como os parâmetros que levaram à escolha dessa amostra de municípios, não são do conhecimento do autor. De todo modo, a amostra cobre um grande número de municípios da Bahia, espalhados por todas as suas regiões, configurando-se, portanto, em uma boa representação da situação do Estado da Bahia. A relação completa dos municípios que compuseram a amostra pode ser encontrada em Nascimento (2003).
} 
capital do Estado e onde vive e estuda boa parte da população baiana, para o qual não havia disponível o IDE (Índice de Desenvolvimento Econômico). Sua exclusão, por outro lado, pode ter evitado a presença de um potencial outlier ${ }^{3}$ no modelo, uma vez que é em Salvador e região metropolitana onde está concentrada a maior parte do PIB da Bahia ${ }^{4}$.

De qualquer forma, o número de observações, que variou de 185 a 235, foi significativo para cada uma das quatro regressões rodadas e está informado na Tabela 1 infra (seção 4).

\section{Identificação das variáveis}

Quanto às variáveis utilizadas, elas foram extraídas de cinco fontes: o Anuário Estatístico da Educação 2000 (BAHIA, 2001), publicação oficial do Governo do Estado da Bahia, o CD-ROM Informações Municipais 2000 (BAHIA, 2002), produzido pelo Tribunal de Contas dos Municípios do Estado da Bahia - TCM, - Censo Demográfico 2000 (IBGE, [2001?]), realizado pelo Instituto Brasileiro de Geografia e Estatísticas - IBGE, o sítio na internet (BAHIA, [199-?]), da Superintendência de Estudos Econômicos e Sociais da Bahia - SEl, e a base de dados sobre desempenho, levantada para o Projeto de Avaliação Externa da Secretaria de Educação do Estado da Bahia $^{5}$, e também utilizada por Azevedo e outros $(2002,2003)$.

Das duas primeiras fontes foram extraídos dados para a construção de quatro variáveis de controle indicativas do volume de recursos educacionais disponíveis, quais sejam:

Gastos efetivos com educação fundamental por aluno (doravante designada pela sigla GASTOS): calculada dividindo o valor efetivamente despendido em educação fundamental pelo município (dado disponível no citado CD-ROM do TCM) pelo número de matrículas iniciais nas escolas do ensino fundamental de sua alçada (dado divulgado pelo Anuário Estatístico da Educação 2000);

Mínimo constitucional (que será aqui indicada pela sigla MINCON): variável binária que assume o valor de 1 quando 0 município alcançou ou superou o percentual mínimo previsto pela Constituição Federal de aplicação por parte dos municípios de $25 \%$ das suas receitas de impostos e transferências no ensino fundamental ${ }^{6}$.

\footnotetext{
${ }^{3}$ Outlier é o termo que se usa em estatística para designar uma observação que destoa sobremaneira das demais por apresentar valores muito superiores ou inferiores à média, podendo gerar, inclusive, vieses ao modelo aplicado. Esta observação em relação a Salvador é pertinente por se tratar de um município que concentra população e renda, mas ainda assim é relativa, pois outros municípios de renda elevada da sua região metropolitana, e, portanto, também potenciais outliers, constam das observações do modelo.

${ }^{4}$ Em 2000, Salvador detinha 18,7\% da população do Estado e respondia por 20\% de seu PIB. A sua região metropolitana (Salvador mais nove municípios), onde estão concentrados a indústria de transformação e o setor de serviços da Bahia, tinha 23,1\% da população do Estado e gerava mais de 50\% do PIB baiano (Fonte: SEI, a partir de dados do Censo 2000 do IBGE).

${ }^{5}$ Agradeço à equipe técnica do Projeto de Avaliação Externa, coordenado por Lys Vinhaes, por ceder os dados para a variável dependente. ${ }^{6}$ Vale ressaltar que do cálculo desses $25 \%$ é excluída uma das transferências constitucionais: o FUNDEF. Como este é um fundo a ser aplicado exclusivamente na educação fundamental, não faz sentido continuar a incluí-lo no montante efetivamente gasto com ensino fundamental quando se faz o cálculo do percentual que esse montante representa do total de receitas provenientes de impostos e transferências. Esta informação foi fornecida por uma fonte do TCM, por telefone. A mesma fonte confirmou que o valor absoluto do dispêndio com educação fundamental informado na tabela extraída do CD-ROM Informações Municipais 2000 inclui a transferência advinda do FUNDEF. Assim, a variável GASTOS compreende o gasto do município em educação fundamental por aluno com recursos provenientes de todas as suas fontes de receita e aplicados em todas as suas modalidades de despesas de custeio e investimento no setor (gastos com pessoal, merenda escolar, transporte, infra-estrutura das escolas, dentre outros).
} 
Proporção de professores em relação ao número de alunos (designada adiante pela sigla DOCENTES/ALUNOS): seu cálculo foi auferido dividindo o número de professores do ensino fundamental da rede municipal pelo número de matrículas iniciais da $1^{a}$ à $8^{a}$ séries (BAHIA, 2001).

Grau de formação dos professores (referida a partir daqui como FORMPROF4 e FORMPROF8, sendo que o número no final de cada sigla informa se a variável utiliza dados para $1^{a}$ a $4^{a}$ séries ou para $5^{a}$ a $8^{\mathrm{a} 7}$ ): variável binária que assume 0 valor 1 quando o município apresenta em seus quadros um percentual de professores com nível superior maior do que a média do Estado e o valor 0 quando ocorre o contrário.

A partir dos dados do IBGE e da SEI, foram adotadas inicialmente nove variáveis de controle para designarem os impactos de condições socioeconômicas e de habilidades pré-adquiridas no desempenho escolar: grau de urbanização do município GRAURB (IBGE, [2001?]), taxa de alfabetização da população de 25 a 49 anos - TXAFPAIS (IBGE, [2001 ?]), Produto Interno Bruto do Município - PIBSEI (BAHIA, [199-?]), rendimento mediano pelo rendimento médio -RMDMÉDIO (IBGE, [2001?]), participação no PIB da região geo-econômica PARTPIB (AZEVEDO et al., 2002) e, a partir de dados da SEI (BAHIA, [199-?]), Índice de Desenvolvimento Econômico - IDE, Índi- ce de Desenvolvimento Social - IDS, Índice de Infra-estrutura - INF, e Índice de Qualificação de Mão-de-obra - IQM. Este grupo de variáveis foi selecionado para designar as condições socioeconômicas e as habilidades pré-adquiridas (cuja proxy utilizada foi TXAFPAIS).

Algumas dessas variáveis foram excluídas por apresentarem altos graus de multicolinearidade com outras, o que pode ser evidenciado pela correlação entre elas. Embora a teoria preveja uma certa correlação entre as variáveis de condições socioeconômicas com as de recursos educacionais e habilidades (UNNEVER; KERCKHOFF; ROBINSON, 2000), correlações altas demais são indícios de multicolinearidade acentuada, o que afeta a qualidade dos testes $†$ para a significância dos coeficientes estimados. PIBSEl foi excluída por sua alta correlação com três outras variáveis: IDE $(0,976587)$, INF $(0,90155)$ e IQM $(0,897696)$. INF9 também o foi pelo mesmo motivo: além de sua alta correlação com PIBSEl, também apresentou elevados graus com IQM $(0,997742)$. IDS, por sua vez, apresentou elevada correlação positiva com GRAURB $(0,802362)$ e $(-0,69325)$ de correlação negativa com RMDMÉDIA.

Outras variáveis precisaram ser eliminadas em razão da heterocedasticidade, fenômeno violador das hipóteses básicas do modelo clássico de regressão linear que ocorre quando a

\footnotetext{
${ }^{7}$ As tabelas que constam da fonte disponível não desagrega essa informação por série, mas por conjunto de séries. Por isso, não foi possível construir a variável FORMPROF apenas com dados sobre a formação dos professores das séries objeto do estudo ora apresentado, isto é, $4^{\mathrm{a}}$ e $8^{\mathrm{a}}$ séries.

${ }^{8}$ A média do Estado da Bahia em 2000, para as quatro primeiras séries, era de 4,03\% dos professores com nível superior completo, ao passo que os professores em exercício da $5^{a}$ a $8^{a}$ séries tinham nível superior completo em 40,24\% dos casos para o ano de 2000.

${ }_{9}$ Pode-se questionar porque INF, e não IQM, foi descartada, sendo que ambas têm correlação alta e muito próxima com duas outras (incluindo entre elas). Essa escolha, de fato, tem um certo grau de subjetividade, embora pautada em fatos objetivos: IQM, que trata da qualificação da mão-de-obra, parece ser mais apropriada a estudos sobre educação do que INF, que mensura infraestrutura do município, a partir de dados como consumo per capita de energia elétrica, número de terminais telefônicos em serviço, número de estabelecimentos bancários em funcionamento, dentre outros.
} 
variância de cada termo de perturbação, condicional aos valores das variáveis de controle, é inconstante, isto é, quando os resíduos não são homogêneos (GUJARATI, 2000; HILL; GRIFFTHS; JUDGE, 2000; MATOS, 1995). Em havendo heterocedasticidade, tem-se como conseqüência que o método dos mínimos quadrados não gera parâmetros eficientes ou de variância mínima. Além do mais, verificam-se erros-padrões enviesados e incorreções dos testes te $\mathrm{F}$ dos intervalos de confiança (MATOS, 1995). Identifica-se a presença de heterocedasticidade ao se analisar os gráficos de resíduos das regressões e ao se aplicar o teste Goldfeld-Quan$\mathrm{dt}^{10}$ a cada uma das variáveis de controle do modelo. Com esses procedimentos, constatouse a presença de heterocedasticidade para as variáveis IDE, IQM e PARTPIBRE, que foram, por conseguinte, eliminadas do modelo.

Assim, foram mantidas as seguintes variáveis de controle ou explicativas: GRAURB, TXAFPAIS, RMDMÉDIA, GASTOS e DOCENTES/ALUNOS, além das variáveis binárias (MINCON, FORMPROF4 e FORMPROF8).

Por fim, a base de dados sobre desempenho, levantada para o Projeto de Avaliação Externa da Secretaria de Educação do Estado da Bahia, foi responsável pela construção das variáveis dependentes: o resultado dos estudantes nos testes de desempenho aplicados pelo Governo do Estado da Bahia. Os mode- los do Projeto de Avaliação Externa basearam-se em testes aplicados a um universo de 41.751 alunos de $4^{a}$ série em 1.564 escolas, enquanto que para a $8^{a}$ série foram 45.764 alunos em 724 escolas para Português e 47.755 alunos em 761 escolas para Matemática. Desses universos foram extraídos os dados usados no modelo ora aplicado, sendo que a unidade de observação aqui utilizada foi o município, ou seja, os dados foram agregados ao nível municipal, variando o número de observações (vide Tabela 1 abaixo) a depender das informações disponíveis para as variáveis explicativas selecionadas.

Assim, as variáveis dependentes das regressões rodadas neste trabalho são o score médio dos alunos da série em questão ( $4^{a}$ ou $8^{a}$, a depender da regressão) das redes de escolas dos municípios integrantes da amostra específica (que varia em tamanho para cada regressão em razão das informações disponíveis para as variáveis explicativas do modelo) nas disciplinas avaliadas (Português ou Matemática, a depender da regressão).

\section{O modelo a ser aplicado}

Portanto, o modelo de regressão linear a ser aplicado na próxima seção é múltiplo, aplicado para dados que reúnem observações em um único ano em um conjunto de municípios baianos, e assume a forma genérica abaixo:

$$
y_{h i j}=\beta_{0}+\sum_{i=1}^{p} \beta_{k} x_{k}+\sum_{i=1}^{p} \delta D_{1}+\sum_{i=1}^{p} \varphi_{k} D_{1} * x_{k}+\sum_{i=1}^{p} \theta D_{2}+\sum_{i=1}^{p} \psi_{k} D_{2} * x_{k}+\varepsilon_{h i j}
$$

Onde:

$\gamma_{\text {hij }}$ é a média do desempenho dos alunos da série $h$ das escolas da rede municipal do município i na prova da disciplina $i ; \beta_{k}$ representa a associação entre a variável $x_{k^{\prime}}$ (que pode ser

\footnotetext{
${ }^{10}$ Ver forma de aplicação detalhada do teste Goldfeld-Quandt em Nascimento (2003) e Gujarati (2000).
} 
GRAURB, TXAFPAIS, RMDMÉDIA, GASTOS e DOCENTES/ALUNOS) e o indicador de desempenho;

$D_{1}$ e $D_{2}$ são as variáveis binárias $M I N$ CON e FORMPROF4, para as regressões aplicadas aos resultados concernentes à $4^{\mathrm{a}}$ série, ou FORMPROF8, para as regressões que tenham como variável dependente o desempenho de alunos de $8^{a}$ série e, por conseguinte, $\delta$ e $\theta$ são as medidas de associação dessas variáveis com $\gamma_{\text {hij }}$; os testes sob a hipótese nula de que $\delta=0$ e $\theta=0$ medem possíveis diferenças de intercepto $\left(\beta_{0}+\delta\right.$ e $\left.\beta_{0}+\theta\right)$, independentemente das demais variáveis que influem no desempenho escolar, enquanto que a diferença de inclinação na associação da variável $x_{k} \operatorname{com} \gamma_{\text {hij }}$ é fornecida por $\varphi_{k}$ e $\psi_{k} i$

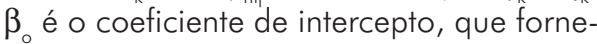

ce a média ou o efeito médio sobre $\gamma_{\text {hij }}$ de todas as variáveis excluídas do modelo; e $\varepsilon_{\text {hij }}$ é o termo de perturbação estocástico do desempenho dos alunos da série $h$ das escolas da rede municipal do município $i$ na prova da disciplina $i$.

\section{Desempenho x recursos: eviência para municípios baianos}

\section{Os resultados das regressões}

Os resultados obtidos com as regressões realizadas surpreendem, contudo, pela ausência de relação significativa entre o rendimento dos estudantes e as variáveis explicativas utilizadas. Para todas as regressões, a estatística $F$ e $\circ R^{2}$ apresentaram coeficientes muito baixos, conforme pode ser atestado na tabela a seguir:

Tabela 1 - Estatísticas das regressões.

\begin{tabular}{|l|c|l|l|l|l|}
\hline REGRESSÃO & Observações & R múltiplo & R Quadrado & $\begin{array}{c}\text { R Quadrado } \\
\text { ajustado }\end{array}$ & Erro Padrão \\
\hline $\begin{array}{l}\text { Regressão 1: 4} \\
\text { série - Português }\end{array}$ & 235 & 0,176487 & 0,0311477 & 0,0012712 & 0,4484228 \\
\hline $\begin{array}{l}\text { Regressão 2: 4 } \\
\text { série - Matemática }\end{array}$ & 235 & 0,1440737 & 0,0207572 & $-0,00944$ & 0,44194 \\
\hline $\begin{array}{l}\text { Regressão 3: 8 } \\
\text { série - Português }\end{array}$ & 185 & 0,3255767 & 0,1060002 & 0,0706442 & 0,4591538 \\
\hline $\begin{array}{l}\text { Regressão 4: 8 } \\
\text { série - Matemática }\end{array}$ & 185 & 0,2174259 & 0,047274 & 0,0095956 & 2,3049546 \\
\hline
\end{tabular}

As estatísticas $\dagger$ das variáveis também foram irrelevantes: a exceção da variável RMDMÉDIA ${ }^{11}$ na regressão 3, nenhuma outra mostrou-se significativa a intervalos de confiança de 95\%, nem mesmo aquelas que representam condições socioeconômi- cas consideradas por ampla maioria da literatura como relevante para o desempenho escolar. As tabelas seguintes mostram, para cada regressão, os valores observados para os coeficientes das variáveis, seus erros-padrão e suas estatísticas t.

\footnotetext{
${ }^{11}$ Seguindo o que seria esperado, RMDMÉDIA apresentou sinal negativo. Como esta variável mede o grau de desigualdade de renda, sendo esta maior quanto maior for o valor que RMDMÉDIA assumir, era de se esperar que seu coeficiente aparecesse com sinal negativo, indicando que quanto melhor for a distribuição de renda do município, melhor o desempenho médio de seus alunos.
} 
Tabela 2 - Coeficientes, erros-padrão e estatísticas $t$ das variáveis explicativas nas regressões para a $4^{a}$ série.

\begin{tabular}{|c|c|c|c|}
\hline \multicolumn{5}{|c|}{ PORTUGUÊS } \\
\hline $\begin{array}{c}\text { VARIÁVEL } \\
\text { intersecção }\end{array}$ & $-0,136365625$ & 0,3136168 & $-0,434816$ \\
\hline GRAURB & $-0,000570499$ & 0,0018585 & $-0,306973$ \\
\hline TXAFPAIS & 0,015153684 & 0,0326043 & 0,4647752 \\
\hline RMDMÉDIA & $-0,480033786$ & 0,2983428 & $-1,609001$ \\
\hline MINCON & 0,080466279 & 0,0695206 & 1,1574458 \\
\hline GASTOS & $-0,000162606$ & 0,0001876 & $-0,866561$ \\
\hline DOCENTES / ALUNOS & 1,008193678 & 4,016217 & 0,2510307 \\
\hline FORMPROF4 & 0,11589586 & 0,1430572 & 0,8101363 \\
\hline VARIÁVEL & MATEMÁTICA & & \\
\hline interSecção & COEFICIENTE & ERRO PADRÃO & ESTAT. $\boldsymbol{t}$ \\
\hline GRAURB & $-0,103143433$ & 0,3090829 & $-0,333708$ \\
\hline TXAFPAIS & $-0,002670225$ & 0,0018316 & $-1,457867$ \\
\hline RMDMÉDIA & 0,005315957 & 0,032133 & 0,1654362 \\
\hline MINCON & $-0,469003464$ & 0,2940297 & $-1,595089$ \\
\hline GASTOS & 0,008427598 & 0,0685155 & 0,1230028 \\
\hline DOCENTES/ALUNOS & $-6,3904 E-05$ & 0,0001849 & $-0,345554$ \\
\hline FORMPROF4 & 1,83226418 & 3,958155 & 0,4629086 \\
\hline & 0,112697332 & 0,1409891 & 0,7993338 \\
\hline
\end{tabular}


Tabela 3 - Coeficientes, erros-padrão e estatísticas $†$ das variáveis explicativas nas regressões para a $8^{a}$ série.

\begin{tabular}{|c|c|c|c|}
\hline \multicolumn{4}{|c|}{ PORTUGUÊS } \\
\hline VARIÁVEL & 0,33180606 & 0,3601943 & 0,9211862 \\
\hline intersecção & $-0,002301802$ & 0,0021193 & $-1,086137$ \\
GRAURB & 0,006522857 & 0,0411048 & 0,1586886 \\
TXAFPAIS & $-1,059153823$ & 0,3375105 & $-3,138136$ \\
RMDMÉDIA & 0,016228384 & 0,0793496 & 0,2045174 \\
MINCON & $4,90753 E-05$ & 0,0002095 & 0,234282 \\
GASTOS & 0,864018622 & 4,5905802 & 0,1882156 \\
DOCENTES/ALUNOS & 0,202843711 & 0,1050241 & 1,9314017 \\
FORMPROF8 & MATEMÁTICA & \\
\hline VARIÁVEL & COEFICIENTE & ERRO PADRÃO & ESTAT. $\mathbf{~}$ \\
\hline interSecção & 50,98854664 & 1,8081774 & 28,198863 \\
GRAURB & $-0,01183392$ & 0,0106387 & $-1,112349$ \\
TXAFPAIS & $-0,147185056$ & 0,2063461 & $-0,713292$ \\
RMDMÉDIA & $-3,021822207$ & 1,6943045 & $-1,783518$ \\
MINCON & $-0,021659074$ & 0,3983356 & $-0,054374$ \\
GASTOS & $-0,000465167$ & 0,0010515 & $-0,442365$ \\
FORMPROF8 & 42,90899512 & 23,044736 & 1,8619869 \\
DONTES/ALUNOS & 0,438548403 & 0,5272215 & 0,8318106 \\
\hline
\end{tabular}

A variável RMDMÉDIA, que pode ser caracterizada como um indicador de distribuição de renda ao exibir a razão entre os rendimentos mediano e médio, parece ter sido a melhor variável explicativa do modelo. Além de estatisticamente significativa para a regressão 3, esteve próximo de sê-lo (e no sinal esperado) em todas as outras regressões. A variável GRAURB, que indica o grau de urbanização do município, apresentou sinal negativo em todas as regressões, o que pode indicar que municípios com menor adensamento urbano obtêm melhor desempenho de seus alunos. Sua estatística $t$, contudo, não foi significativa em nenhuma de- las, embora se tenha aproximado de sê-lo nas regressões 2, 3 e 4 .

Dentre as variáveis de recursos educacionais, a variável binária MINCON (que assume valor 1 quando o município alcança ou supera o percentual mínimo exigido pela Constituição de aplicação de recursos no ensino fundamental) aproximou-se de alguma significância na regressão 1 e no sinal esperado (cumprir o mínimo de 25\% de aplicação de receitas de impostos e transferências na educação afetando positivamente o desempenho). DOCENTE/ALUNO, que exprime a razão do número de professores 
pela de alunos, esteve próxima da significância na regressão 4, o que pode ser indício de que classes menores seriam mais apropriadas para o ensino de Matemática na $8^{a}$ série. Por fim, na regressão 3 a estatística $\dagger$ da variável binária FORMPROF8, que retrata o grau de formação dos professores, esteve muito próxima de 2 (valor mínimo para que a variável seja tomada como significativa), o que poderia ser uma indicação de que o nível educacional dos professores seja importante para o ensino de Português para alunos de $8^{a}$ série.

Ainda que façamos essas elucubrações, todavia, o fato é que os resultados do modelo foram pífios, o que merece uma avaliação mais aprofundada dos resultados.

\section{Análise dos resultados}

Os resultados apresentados na seção 4.1 foram demasiadamente exíguos para as pretensões do presente trabalho. Os baixos valores de parâmetros como a estatística $F$, o $R^{2}$ e as estatísticas + sugerem que o modelo montado não é bom. Por que isto aconteceu?

Algumas explicações podem ser aventadas.

\section{- Razões de ordem econométrica}

Algumas dessas explicações dizem respeito a problemas de especificação do modelo.

A primeira delas é a de que variáveis importantes possam ter sido omitidas. $\bigcirc$ alto valor de bo (coeficiente de intercepto) na Regressão 4 fortalece esta hipótese, uma vez que é ele que informa a influência média das variáveis excluídas do modelo sobre a variável dependente (GUJARATI, 2000).
Em termos genéricos, as conseqüências da omissão de uma variável relevante são: parâmetros enviesados e inconsistentes, caso a variável omitida tenha correlação com as variáveis incluídas, sendo que esse viés não desaparece por maior que seja a amostra; caso não haja correlação com as variáveis incluídas, o coeficiente de intercepto ainda é enviesado, embora os coeficientes de regressão parcial não o sejam; a variância da perturbação $s^{2}$ é estimada incorretamente; as variâncias das estimativas dos coeficientes de regressão parcial tal como são convencionalmente medidas $\left(=s^{2} /{ }^{\circ} X^{2} n i\right)$ são estimadores enviesados das variâncias dos seus verdadeiros estimadores; por conseguinte, os costumeiros intervalos de confiança e testes de hipótese provavelmente darão conclusões enganosas no que tange à significância estatística dos parâmetros estimados (GUJARATI, 2000).

Diante do exposto, poder-se-ia pensar que algumas variáveis importantes tenham sido excluídas quando se fez a análise de correlação (ver Metodologia). Entretanto, ao incluílas uma a uma no modelo, a estatística $F$ e o $R^{2}$ não variam muito. Isto não elimina a possibilidade de haver o viés descrito, tendo em vista que outras variáveis não identificadas podem ser relevantes e terem correlação com aquelas incluídas. Se não existir tal correlação, ainda assim o coeficiente de intercepto continuaria enviesado e seria de se supor então que as variáveis incluídas no modelo são, pelo menos, em sua maioria, irrelevantes. Caso a omissão de variável relevante tenha de fato ocorrido, nada se poderá afirmar acerca da influência no desempenho escolar das variáveis incluídas no modelo, pois os intervalos de confiança e os testes de hipótese darão decerto conclusões enganosas no tocante à significância estatística dos parâmetros estimados. 
O problema ocasionado por uma eventual omissão de variáveis relevantes pode ter sido agravado ainda pela agregação dos dados trabalhados. De acordo com Hanushek, Rivkin e Taylor (1996), quanto maior é o grau de agregação dos dados, maior tende a ser o viés proporcionado pelas variáveis importantes que foram omitidas, porque elas teriam seu efeito mais claro quando os dados estão agregados no nível dos fatores omitidos. Assim, este problema poderia ter sido reduzido se a agregação dos dados fosse ao nível do estudante, ou ao menos da escola, pois seria mais difícil de negligenciar fatores referentes a esses níveis de agregação. A tese de Hanushek, Rivkin e Taylor (1996), todavia, é de que nível de agregação mais elevado gera um viés para cima nos parâmetros relativos a recursos educacionais, o que não parece ter sido o caso $^{12}$.

Outra explicação para o pouco poder de explicação do modelo econométrico aqui utilizado poderia ser a inclusão de uma variável relacionada à renda familiar (que seria a variável RMDMÉDIA), se o argumento de Dewey, Husted e Kenny (2000), segundo o qual esse tipo de variável seria de funções de demanda por educação, e não de produção, fosse acolhido. Porém, exercícios feitos eliminando-se RMDMÉDIA e até mesmo GRAURB não melhoraram a situação, tendo apenas tornado o intercepto significativo nas regressões em que ele antes não era e mais significativo ainda na Regressão 4, na qual esse coeficiente já era bastante significativo.

Tampouco houve êxito na tentativa de se estimar o modelo adicionando aos pou- cos as variáveis, ao invés de usá-las simultaneamente, de acordo com a metodologia descrita em Braga e Guimarães (1986).

Diante disto, a hipótese de que houve omissão de variável relevante ganha mais força, o que configuraria um erro de especificação. Este tipo de erro e o viés que proporciona, em certa medida comuns em modelos econométricos, acontecem "talvez devido à nossa incapacidade de formular o modelo tão precisamente quanto possível, porque a teoria que fundamenta é fraca ou por não termos o tipo certo de dados para testar o modelo" (GUJARATI, 2000, p. 462). Mesmo tendo-se em vista que nem todas as conclusões encontradas pela literatura especializada sejam necessariamente extensíveis ao Brasil e à Bahia, não há porque acreditarmos que a teoria que embasou o modelo deste trabalho tenha sido fraca, visto que tem sido formulada e testada por economistas diversos em variadas partes do mundo e aplicada com sucesso a amostras bem diferentes. $\bigcirc$ que há são dissensos na literatura sobre o tema, já apontados na seção 2 supra. Entretanto, os resultados ora encontrados nem validaram alguma das correntes apresentadas, nem tiveram força suficiente para negar nenhuma delas. Talvez fosse então o caso de os dados não terem sido os mais adequados para testar o modelo, ainda que tenham sido os disponíveis.

Em todo caso, não adianta formular hipóteses acerca do porquê tenha ocorrido eventual omissão de variáveis relevantes, nem ficar explicitando as conseqüências disso se não for averiguado se realmente ocorreu tal erro de especificação.

\footnotetext{
${ }^{12}$ De qualquer forma, não se pode deixar de reconhecer a conveniência da desagregação dos dados, à medida que a distribuição de recursos, de habilidades e de condições socioeconômicas não é uniforme dentro de cada município (AZEVEDO et al., 2003).
} 


\section{Testando se houve omissão de variáveis relevantes}

Uma maneira de testar a hipótese de variáveis importantes terem sido omitidas é por intermédio da estatística d de Durbin-Watson. Esta estatística é simplesmente a razão entre a soma das diferenças ao quadrado nos sucessivos resíduos e a soma dos quadrados dos resíduos, sendo definida pela forma matemática abaixo (GUJARATI, 2000):

$$
d=\sum_{t=2}^{t=n}\left(\hat{u}_{t}-\hat{u}_{t-1}\right)^{2} / \sum_{t=2}^{t=n} \hat{u}_{t}^{2}
$$

Utilizada originalmente para detectar a correlação serial, a estatística d de DurbinWatson também pode ser usada para detectar eventuais erros de especificação, devendo-se, para tanto, seguir o seguinte procedimento (GUJARATI, 2000):

(1) Do modelo adotado por hipótese, obter-se os resíduos por mínimos quadrados ordinários;

(2) Caso se acredite que o modelo adotado por hipótese esteja incorretamente especificado porque exclui uma variável explicativa $Z$ relevante, ordenar os resíduos obtidos em (1) segundo os valores crescentes de $Z$, sendo que esta variável hipotética pode ser uma das variáveis incluídas no modelo adotado por hipótese ou alguma função dela;

(3) Calcular a estatística d dos resíduos assim ordenados através da fórmula usual de $d$, apresentada acima;

(4) Com base nas tabelas de DurbinWatson, se o valor estimado de $d$ for significativo, pode-se então aceitar a hipótese de erro na especificação do modelo.
A averiguação da significância do valor estimado de d se dá de uma maneira peculiar. Como é calculada a partir dos resíduos, esta estatística depende dos valores dados da variável de controle utilizada no teste. Por isso, ao contrário dos testes $t$, $F$ ou $c^{2}$, não há um valor crítico único que permita concluir pela rejeição ou pela aceitação da hipótese nula de que não houve erro de especificação. $O$ que há é um intervalo de valores críticos. Quando o valor calculado de $d$ for menor que o limite inferior desse intervalo, conclui-se pela ocorrência do erro. Quando for maior que o limite superior, não há indícios de que variáveis relevantes foram omitidas. Se esse valor ficar dentro do intervalo crítico, não há subsídios para se afirmar se houve ou não a omissão (GUJARATI, 2000).

No caso em tela, o teste d de DurbinWatson mostrou indícios de omissão de variáveis relevantes para as regressões válidas para a $8^{a}$ série (regressões 3 e 4). Os valores calculados de $d$ para as regressões relativas à $4^{a}$ série (regressões 1 e 2) estiveram acima do limite superior do intervalo de valores críticos dessa estatística, não apresentando, portanto, quaisquer indícios de omissão de variáveis relevantes.

Assim, o que se pode dizer de concreto é que as variáveis utilizadas não foram capazes de formar um modelo que explicasse a contento o rendimento dos estudantes nas provas aplicadas à $8^{a}$ série. Isto teria ocorrido em razão de omissão de variáveis relevantes, cuja identificação não foi possível fazer, pois mesmo testando-se regressões com diferentes proxies para os fatores identificados pela literatura como determinantes do desempenho escolar, o resultado foi pífio. 
É possível também que os dados disponíveis para a montagem das variáveis não tenham tido qualidades suficientes para figurarem como proxies apropriadas dos fatores determinantes do desempenho escolar. Isto é particularmente válido para as proxies de características socioeconômicas, uma vez que a maioria delas teve que ser excluída, quer por apresentar correlação com outras, quer por gerar heterocedasticidade, e de habilidades inatas dos estudantes, que só pôde ter como proxy a taxa de alfabetização da população adulta dos municípios, que informa muito pouco sobre o grau de formação escolar dos pais dos alunos ${ }^{13}$. No caso das variáveis de recursos educacionais, o fato de não haver sido possível mensurar diferentes tipos de gastos pode também ter prejudicado, ainda que não de tamanha magnitude a ponto de comprometer o modelo. Não havia como medir tampouco os efeitos da corrupção sobre a efetiva aplicação desses recursos.

Não obstante as razões de ordem econométrica apresentadas para a diminuta colaboração prática das regressões rodadas, uma pergunta persiste: por que não se conseguiu extrair da amostra com municípios baianos utilizada resultados que fizessem das redes municipais de ensino fundamental da Bahia um exemplo empírico da tese de alguma das correntes da literatura econômica sobre o tema?

A resposta para tal pergunta decerto remete a razões de ordem socioeconômica e cultural.

\section{- Razões de ordem socioeconômica e cultural}

A primeira delas é que alunos que dispõem de melhores condições socioeconômicas teriam capacidade maior de traduzir os insumos educacionais aplicados em sua educação em melhores resultados, isto é, maximizariam suas funções insumo-produto da educação. Como as observações da amostra não tinham uma variação significativa das características socioeconômicas (com exceção das variáveis GRAURB e GASTOS, as variâncias estimadas foram bem pequenas), não teria sido possível detectar isto no modelo usado.

Neste sentido, é pertinente lembrar algumas das hipóteses levantadas por Unnever, Kerckhoff e Robinson (2000): a de que as populações de localidades com status socioeconômico mais elevado dispõem de melhores recursos educacionais e a de que localidades com melhores recursos educacionais obtêm melhores desempenhos de seus estudantes, mesmo controlando os níveis de habilidade individuais dos estudantes e as características socioeconômicas locais. Esses recursos não seriam somente aqueles oferecidos diretamente pelas escolas, mas também aqueles tornados disponíveis ao estudante pela sua própria família, como, por exemplo, o computador e a biblioteca que existem em casa e o curso de línguas em escola especializada que os pais pagam aos filhos.

Não há como comprovar isto para a Bahia com a amostra ora utilizada, nem tampouco é este o escopo do presente trabalho. Ainda

\footnotetext{
${ }^{13} \mathrm{O}$ ideal para mensurar o fator habilidades pré-adquiridas pelos estudantes seria uma série histórica da média dos desempenhos dos mesmos estudantes em testes de aproveitamento anteriores, o que não foi possível por não se dispor desses dados. Pensou-se, então, em colocar como variável a média do número de anos de estudos da população adulta de cada município, dado que não foi encontrado, daí a necessidade de ser recorrer somente à taxa de alfabetização da população adulta.
} 
assim, isto parece ser verdade quando se visualiza a realidade e a composição do corpo discente de escolas públicas e privadas Bahia afora. Caso o seja, um outro ponto aportaria na questão da qualidade da educação e na equalização de oportunidades no Estado, podendo ser objeto de futuros trabalhos: a constatação de um ciclo vicioso da heterogeneidade educacional, segundo o qual as populações com status socioeconômico mais elevado, por terem acesso a melhores recursos educacionais, obterão sempre desempenhos mais elevados de seus estudantes do que as populações de status socioeconômico menor, a não ser que o Poder Público destine os seus melhores recursos às populações de menor status, quebrando, assim, esse ciclo.

Outra possível razão para o reduzido poder explicativo do modelo aplicado seriam os valores observados para a própria variável dependente. $\bigcirc$ desempenho dos alunos mostrou-se nivelado por baixo em todas as provas. A variância das notas foi pequena e a média se situou sempre em patamares baixos para os quatro casos de avaliação (principalmente para o desempenho dos alunos de $4^{a}$ série). Este fato aponta para uma generalizada baixa qualidade do ensino das escolas da rede municipal por todo o território baiano, que poderia ser causada não apenas por fatores diretamente ligados à escola e seu gerenciamento $^{14}$, mas também por grandes limitações de natureza socioeconômicas a que as populações pobres (em regra demandantes das redes públicas de ensino) estão sujeitas na quase totalidade dos municípios baianos, principalmente nas regi- ões rurais, limitações estas que vão desde carências alimentares e ausência de saneamento básico até dificuldade de acesso (transporte) à escola.

Além disso, não seria errado supor que os alunos que se submeteram aos testes não tinham motivação para respondê-los empreendendo seus melhores esforços, uma vez que o resultado de seus desempenhos não iria influenciar na aprovação naquele ano em suas escolas, nem tampouco senviria de pré-requisito para que eles auferissem eventuais vantagens diretas imediatas. Embora este tipo de comportamento cause prejuízos à formulação de políticas educacionais que visem a melhorar a qualidade do ensino e, por conseqüência, ao próprio estudante, não se pode negar que os que assim agiram o fizeram de maneira racional, pois decerto não tinham maturidade nem orientação suficientes para perceber a importância de dedicar-se àquelas provas e assim podem ter preferido destinar o tempo e o esforço que seriam utilizados na resolução mais apurada das questões das provas em outras atividades que parecessem lhes proporcionar maior bem-estar. Também é possível que nunca tenham sido de fato ensinados os conteúdos testados, indicando uma defasagem entre o currículo mínimo proposto pela Secretaria de Educação e a realidade da sala de aula na rede desses municípios.

Finalmente, vale ressaltar que muitas das conclusões da literatura econômica para o tema aqui explorado podem não ter aplicabilidade geral (HEYNEMAN; LOXLEY, $1982,1983)^{15}$. Afinal, o que se verifica na realidade americana pode não ser extensí-

\footnotetext{
${ }^{14}$ A questão do gerenciamento, por sinal, pode ser decisiva para tornar uma escola mais eficiente do que uma outra. Escolas com o mesmo volume de recursos podem apresentar padrões de qualidade diferentes em função da atuação de seus diretores, ainda que as diretrizes gerais do sistema educacional sejam as mesmas para ambas. O nível de agregação deste trabalho, todavia, não permitiu que diferenças como essas fossem constatadas, por serem reveláveis somente com dados agregados ao nível da escola. ${ }^{15}$ Vide penúltimo parágrafo da seção "Consensos e dissensos...".
} 
vel à realidade brasileira, bem como o que se constata ser como relevante na determinação do desempenho dos alunos sul-coreanos pode não o ser para o desempenho escolar de crianças africanas e assim por diante. Nem mesmo dentro de um mesmo país o ambiente do estudante é o mesmo nas mais diversas regiões, o que no caso do Brasil pode ser comprovado pelos resultados diferentes para cada região encontrados pelo Instituto Nacional de Estudos e Pesquisas Educacionais (INEP, 2003) ${ }^{16}$ em recente publicação quando observa o número de anos que os estudantes levam em média para concluir a educação fundamental e qual o percentual daqueles que não a concluem, em cada região.

\section{Gastos públicos em educação importam?}

Infelizmente, os resultados encontrados no presente trabalho não permitem a formulação de respostas contundentes à pergunta que serve de título a esta seção e que foi o objeto principal deste artigo.

Apesar de ter apresentado coeficientes muito baixos e sinal incerto em todas as quatro regressões, a variável GASTOS não pode ser considerada ineficaz para a qualidade da educação pública dos municípios baianos apenas com base neste trabalho. Isto porque o modelo aplicado não mostrou grande poder explicativo, o que pode ser evidenciado pelos baixos valores que receberam a estatística $F$ e $\circ R^{2}$ nas regressões rodadas.

Nenhuma das outras proxies para recursos educacionais tampouco se mostrou relevante neste modelo, embora MINCON (mínimo constitucional), FORMPROF8 (formação dos professores da $8^{a}$ série) e DOCENTES/ALUNOS (razão do número de professores pelo de alunos) tenham apresentado coeficientes com valores próximos de serem significativos nas regressões 1 ( $4^{a}$ série - Português), 3 ( $8^{a}$ série - Português) e 4 ( $8^{a}$ série - Matemática), respectivamente.

Duas das quatro regressões utilizadas mostraram indícios de omissão de variáveis relevantes, o que gera um viés nos coeficientes nelas estimados. Tal omissão pode ser atribuível à pouca adequação da maior parte dos dados disponíveis à natureza do fenômeno estudado. Além do mais, condições socioeconômicas relativamente homogêneas verificadas na maioria dos municípios analisados, e um possível comportamento de minimização de esforço por parte dos estudantes diante das avaliações de aproveitamento aplicadas podem também ter dificultado uma mensuração apurada do impacto no desempenho escolar das variáveis incluídas no modelo. Há ainda de se levar em conta que o Brasil, e especificamente a Bahia, ainda carecem de estudos como este e, possivelmente, muitas das conclusões da literatura especializada não sejam aplicáveis às suas realidades.

Mesmo assim, tal como na maior parte dos estudos sobre o tema, as características socioeconômicas foram as que mais indicaram influir no desempenho, com a variável RMDMÉDIA (razão do rendimento mediano pelo médio) exibindo sempre o sinal esperado e aparecendo com influência significativa no desempenho em Português dos alunos da

\footnotetext{
${ }^{16}$ Essa publicação é a Geografia da Educação Brasileira 2001, divulgada em março de 2003 pelo INEP e que mostra que 59\% dos alunos brasileiros conseguem completar o nível fundamental de ensino (até a $8^{a}$ série), levando em média 10,2 anos para fazêlo. Esses números mudam a depender da região do País, sendo a pior situação a da região Norte.
} 
$8^{a}$ série. Mesmo sendo este resultado insuficiente para se afirmar que seja este o fator mais determinante do desempenho escolar, não só porque só foi significativo em apenas uma das quatro regressões rodadas, mas também, vale frisar mais uma vez, em conseqüência do baixo poder explicativo demonstrado pelo modelo econométrico utilizado, não se podem desprezar os indícios que esta variável forneceu, no sentido de que desigualdade de renda maior se reflete em heterogeneidade educacional também maior.
De qualquer maneira, a pesquisa científica na Bahia e no Brasil ainda está engatinhando no que se refere a estudos empíricos que visem a mensurar objetivamente os fatores determinantes do desempenho escolar para, assim, levantar subsídios que colaborem para a formulação de políticas públicas mais eficazes para a redução do fosso educacional existente no País e, por conseqüência, das desigualdades que gera e a partir das quais é gerado tal fosso.

\section{Referências}

AKSOY, T.; LINK, C. A panel analysis of student mathematics achievement in the US in the 1990s: does increasing the amount of time in learning activities affect Math achievement? Economics of Education Review, Cambridge, Mass., v. 19, n. 3, p. 261-277, June 2000.

ALEXANDER, N. The missing link: an econometric analysis on the impact of curriculum standards on student achievement. Economics of Education Review, Cambridge, Mass., v. 19, n. 4, p. 351-361, Oct. 2000.

AZEVEDO, J. S. G. et al. Análise multivariada de indicadores de desempenho escolar: impactos de variáveis socioeconômicas: relatório I. Salvador: CME/FCE/UFBA, 2002. Mimeografado.

Análise multivariada de indicadores de desempenho escolar: impactos de variáveis socioeconômicas: relatório II. Salvador: CME/FCE/UFBA, 2003. Mimeografado.

BAHIA (Estado). Secretaria da Educação. Avaliação de desempenho do ensino fundamental do Estado da Bahia: Projeto de Avaliação Externa. Salvador, 2001. BAHIA (Estado). Secretaria da Educação. Superintendência de Estudos Econômicos e Sociais. Anuário estatístico da educação 2000. Salvador, 2001.

BAHIA (Estado). Secretaria da Educação. Superintendência de Estudos Econômicos e Sociais. SEl: informação a serviço da sociedade. Disponível em: <www.sei.ba.gov.br>. Acesso em: 6 ago. 2007.

BAHIA (Estado). Tribunal de Contas dos Municípios do Estado da Bahia. Informações municipais 2000. Salvador, v. 5, n. 5, out. 2002. 1 CD-ROM.

BAKER, D. P.; GOESLING, B.; LETENDRE, G. K. Socioeconomic status, school quality, and national economic development: a cross-national analysis of the "Heyneman-Loxley Effect" on Mathematics and Science achievement. Comparative Education Review, Chicago, v. 46, n. 3, Aug. 2002. 
BETTS, J. Do school resources matter only for older workers? The Review of Economics and Statistics, Cambridge, Mass., v. 78, n. 4, p. 638-652, Nov. 1996.

BETTS, J.; SHKOLNIK, J. The effects of ability grouping on student achievement and resource allocation in secondary schools. Economics of Education Review, Cambridge, Mass., v. 19, n. 1, p. 1-15, Feb. 2000a.

BETTS, J.; SHKOLNIK, J. Key difficulties in identifying the effects of ability grouping on student achievement. Economics of Education Review, Cambridge, Mass., v. 19, n. 1, p. 21-26, Feb. 2000b.

BRAGA, H; GUIMARÃES, E.. Estrutura industrial e exportação de manufaturados no Brasil. Pesquisa e Planejamento Econômico, Rio de Janeiro, v. 16, n. 1, abr. 1986. BRASIL. Constituição (1988). Constituição da República Federativa do Brasil. Brasília, DF: Senado Federal, 1988.

BUCHEL, F.; DUNCAN, G.. Do parent's social activities promote children's school attainment? Evidence from German socioeconomic panel. Journal of Marriage and the Family, Menasha, Wis., v. 60, p. 95-108, 1998.

CARD, D.; KUEGER, A. B.. School resources and student outcomes: an overview of the literature and new evidence from North and South Carolina. The Journal of Economic Perspectives, Nashville, TN, v. 10, n. 4, p. 31-50, 1996.

DEWEY, J.; HUSTED, T.; KENNY, L.. The ineffectiveness of school inputs: a product of misspecification? Economics of Education Review, Cambridge, Mass., v. 19, n. 1, p. 27-45, Feb. 2000.

DRISCOLL, D.; HALCOUSSIS, D.; SVORNY, S.. School district size and student performance. Economics of Education Review, Cambridge, Mass., v. 22, n. 2, p. 193-201, Apr. 2003.

FIGLIO, D. Functional form and the estimated effects of school resources. Economics of Education Review, Cambridge, Mass., v. 18, n. 2, p. 241-252, Apr. 1999.

GUJARATI, D. Econometria básica. São Paulo: Makron Books, 2000.

HANUSHEK, E. The economics of schooling: production and efficiency in public schools. Journal of Economic Literature, Nashville, TN, v. 24, p. 1141-1177, 1986.

HANUSHEK, E.; RIVKIN, S.; TAYLOR, L.. Aggregation and the estimated effects of school resources. The Review of Economics and Statistics, Cambridge, Mass., v. 78, n. 4, p. 611-627, Nov. 1996.

HEDGES, L.; LAINE, R. D.; GREENWALD, R.. Does money matter? A meta-analysis of the effects of differential schools inputs on student outcomes. Educational Researcher, Washington, DC, v. 23, n. 3, p. 5-14, Apr. 1994.

The effect of school resources on student achievement. Review of Educational Research, Washington, DC, v. 66, p. 361-396, 1996 a.

. Interpreting research on school resources and student achievement: a rejoinder to Hanushek. Review of Educational Research, Washington, DC, v. 66, p. 411-416, $1996 \mathrm{~b}$. 
HEYNEMAN, S.; LOXLEY, W. The effect of primary school quality on academic achievement across twenty-nine high- and low-income countries. American Journal of Sociology, Chicago, v. 88, n. 6, p. 1162-94, 1983.

HEYNEMAN, S.; LOXLEY, W. Influences on academic achievement across high and low income countries: a re-analysis of IEA data. The Sociology of Education, London, v. 55 n. 1, p. 13-21, 1982.

HILL, C.; GRIFFITHS, W.; JUDGE, G. Econometria. São Paulo: Saraiva, 2000.

IBGE. Disponível em: <http://www.ibge.gov.br>. Acesso em: 15 out. 2002.

INEP. Geografia da educação brasileira 2001. Brasília, 2003. Disponível em: <www.inep.gov.br>. Acesso em: 13 mar. 2003.

JONES, J.; ZIMMER, R. W.. Examining the impact of capital on academic achievement. Economics of Education Review, Cambridge, Mass., v. 20, n. 6, p. 577-588, Dec. 2001. $\mathrm{KIM}, \mathrm{H}$..Is there a crowding-out effect between school expenditure and mother's child care time? Economics of Education Review, Cambridge, Mass., v. 20, n. 1, p. 71-80, Feb. 2001.

LAVY, V. Disparities between Arabs and Jews in school resources and student achievement in Israel. Economic Development and Cultural Change, Chicago, v. 47, n. 1, p. 175-192, 1998.

MCEWAN, P. Peer effects on student achievement: evidence from Chile. Economics of Education Review, Cambridge, Mass., v. 22, n. 2, p. 131-141, Apr. 2003.

MATOS, O. C. Econometria básica: teoria e aplicações. São Paulo: Atlas, 1995. NASCIMENTO, P. A. M. M. Desempenho escolar e gastos municipais por aluno em educação: relação observada em municípios baianos. 2003. 70 f. Trabalho de Conclusão de Curso (Graduação em Economia)-Universidade Federal da Bahia, Salvador, 2003.

SANDER, W. Expenditures and student achievement in Illinois. Journal of Public Economics, Amsterdam, v. 52, p. 403-416, 1993.

UNNEVER, J.; KERCKHOFF, A.; ROBINSON, T. District variations in the educational resources and student outcomes. Economics of Education Review, Cambridge, Mass., v. 19, n. 3, p. 245-259, June 2000.

Recebido em: 07/1 1/2006

Aceito para publicação em: 04/04/2007 